\title{
Research on Application of Internet of Things and Cloud Computing Technology in Intelligent Medical System
}

\author{
Zijia Chen1,2,a,Zhenghong Guo1,2,b ,Xin Liu1,2,c and Xiao Zhang1,3,d *
}

1School of Information Science and Engineering, Hebei North University, Zhangjiakou075000, China

2Medical Informatics in Hebei Universities Application Technology Research and Development Center,Zhangjiakou075000, China

3Population Health Informatization in Hebei Province Engineering Technology Research Center,Zhangjiakou075000, China

Aczjiahb22@163.com ,bzjkgzh@sina.com,cliux92012@126.com,dchenxiao2232@yeah.net

* The corresponding author

Keywords: Intelligent medical; Internet of things; Medical big data; Intelligent perception; RFID

\begin{abstract}
The new information technology is the key technology to meet the demand of medical services, and medical wisdom is an important research direction of medical information. It is the fusion of networking, cloud computing and big data processing technology, which can greatly improve the medical quality, and improve the efficiency of management of the hospital medical object, rational allocation and utilization of medical resources.
\end{abstract}

\section{Introduction}

Wisdom medicine is a kind of new medical form in the era of Internet of things[1]. The concept originated in the development of Internet of things. The basic idea of the Internet of things was proposed in the late 1990s, and the source is RFID system, which was proposed by network of radio frequency identification in automatic identification center of Massachusetts Institute of Technology (MIT), that is, connect all of the matters to Internet through the radio frequency identification information sensing equipment, realizing intelligent identification and management. The Internet of things is widely used in public security, urban management, intelligent transportation, environmental monitoring, remote medicine and other fields[2], while Wisdom medicine is the product of the Internet of things in the field of medical applications. In 1994, the World Telecommunication Development Conference proposed to enhance the development of medical services in developing countries by using information and communication services[3]. In 2005, the World Health Assembly held the point that digital health care could achieve low-cost, efficient services, and stressed that Member States actively engaged in digital medical planning[4]. In 2007, the International Telecommunication Union issued guidelines and principles for developing digital health care in developing countries[5]. In 2008, IBM further puts forward the concept of Wisdom Earth, then the Internet of things technology will be applied to the medical field, realizing Internet sharing of medical information. At the same time, the IOT technology helps to integrate medical platform and electronic health records system.

\section{Application of Internet of Things in Wisdom Medicine}

In the "12th Five-Year" plan, networking was planned for the medical and health system, which not only can help the hospital to monitor the health of patients, such as implantation of chip into patient body, monitoring various indexes and giving proper advice at any time, but also carry out the effective management of the hospital's operation, in order to realize implementation of intelligent and humanized management towards hospital staff, equipment, logistics supply, traffic safety. This not 
only effectively saves social resources, but also greatly promote the operating speed of the medical and health system. The following is an example to illustrate the application of intelligent medicine.

Taking production inspection as an example. Assuming that a pregnant woman needs 12 times inspections from pregnancy to the production, then throughout the pregnancy, the pregnant woman have to go back and forth from home to the hospital at least 12 times. In fact, most of the routine examinations of pregnant women can be completed by the instruments to complete. If wisdom medical model was used, the pregnant women only need to go to the hospital for check in the first and last production, and can be completed independently at home in any other time, and transmit information to the hospital by the doctor to make the inspection report.

From this simple example we can see that the wisdom medical model has advantages of saving time to see a doctor, saving the medical resources in general. Compared with traditional medical wisdom medical service model, wisdom medical mainly has the following advantages. Automatic or self collection of various types of human vital signs data by using a variety of sensors and medical for family can reduce the medical personnel burden, and can obtain abundant data more frequently. The collected data is automatically transmitted to the hospital data center through the wireless network, and the medical staff can use the data to provide remote medical service. Centralized storage management data can achieve data sharing and depth utilization, so as to analyze and mine on a large number of medical data, and help resolve key medical records and difficult miscellaneous diseases. In addition, it can lower the cost of sub healthy people, the elderly and patients with chronic diseases to provide long-term, rapid and stable health monitoring and treatment services, and reduce risk, which indirectly reduce scarce medical resources demand, for example ,as shown in fig. 1.

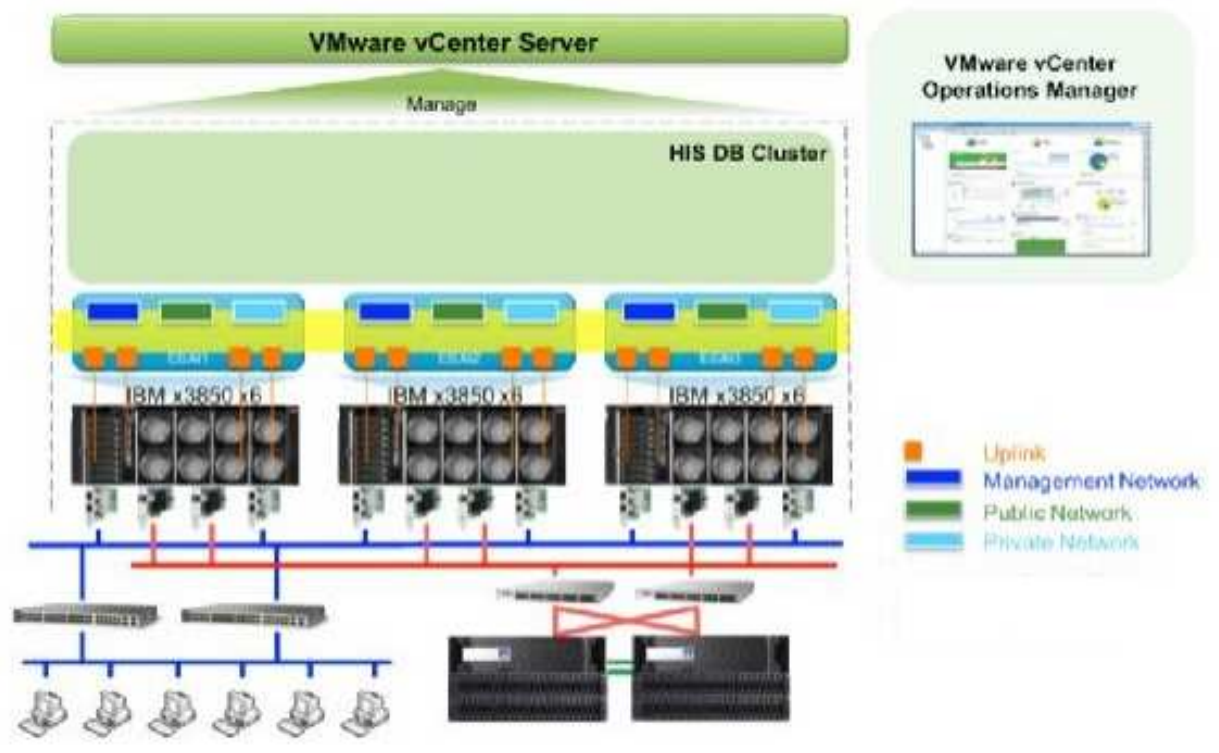

Figure 1. The topology of intelligent medical project hospital

\section{Wisdom Medical System based on Internet of Things and Cloud Computing}

Medical Networking. The key to the realization of the wisdom medical are Internet of things technology and cloud computing technology, and the connection point of these two technologies is a huge amount of medical data, or can be referred to as medical big data. In medical Internet of things, all kinds of sensors and medical devices produce various data, these data in large scale, rapid growth rate, the traditional database technology has been unable to effectively carry on the management and processing. So in wisdom medical, the introduction of cloud computing technology, dedicated to medical services to a cloud computing platform with low cost can achieve efficient and scalable medical data storage, and can provide convenient medical services to users through the internet. 
Different from the existing medical information system, wisdom medical and depth data acquisition is emphasized by the extensive data collection, namely the use of various means is not affected by the types of data collected time and location constraints. Although the existing electronic medical records system can save all the patients in the hospital for examination and medical information, the data is very limited. Intelligent medical use Internet of things technology at any time and place to collect a variety of human vital signs data and automatically save, the amount of data is much larger than the amount of data recorded manually into the electronic medical records. The depth of data, namely discover hidden knowledge from data by using data mining and machine learning techniques, such as changes of oxygen saturation period, abnormal heart rate detection, and vital signs related to change mode. Due to the referred type of data is various and with large scale, these knowledge is hard to be got by a doctor with experience in an artificial way. In addition, the application of large-scale data processing technology can also analyze all the records of patients, and help doctors resolve difficult miscellaneous diseases.

Core Idea of Medical Internet of Things. The core of health care reform is to realize whole process of health management. Effective means of medical networking is an important support for wisdom medical. The core content of medical wisdom includes intelligent perception of medical objects, standardized disposal of medical procedures through the interconnection technology, to realize the integration of medical objects and medical process, standardization management of the whole process of medical objects, so as to achieve the goal of improvement of medical safety and medical quality.

Digital hospital management medical card is a comprehensive application of smart card in the hospital. It is used by all employees working in the hospital, and used for the patient life from all aspects, including personnel information management, outpatient and emergency management, hospital management, consumption management, apartment management. Moreover, it is not only the carrier of cardholder information management, but also the important facilities of hospital logistics services. The intelligent health care label is placed on the RFID card, and the tag can complete their medical records of patients. In addition, RFID cards are for each staff to wear, one hand is used to identify the medical staff's identity, on the other hand, the card also integrated RFID reader function, and the patient's treatment through the built-in wireless communication card any antenna. Any doctor or other medical personnel can timely read and store important disease information. The communication architecture of digital hospital management is shown as fig. 2 .

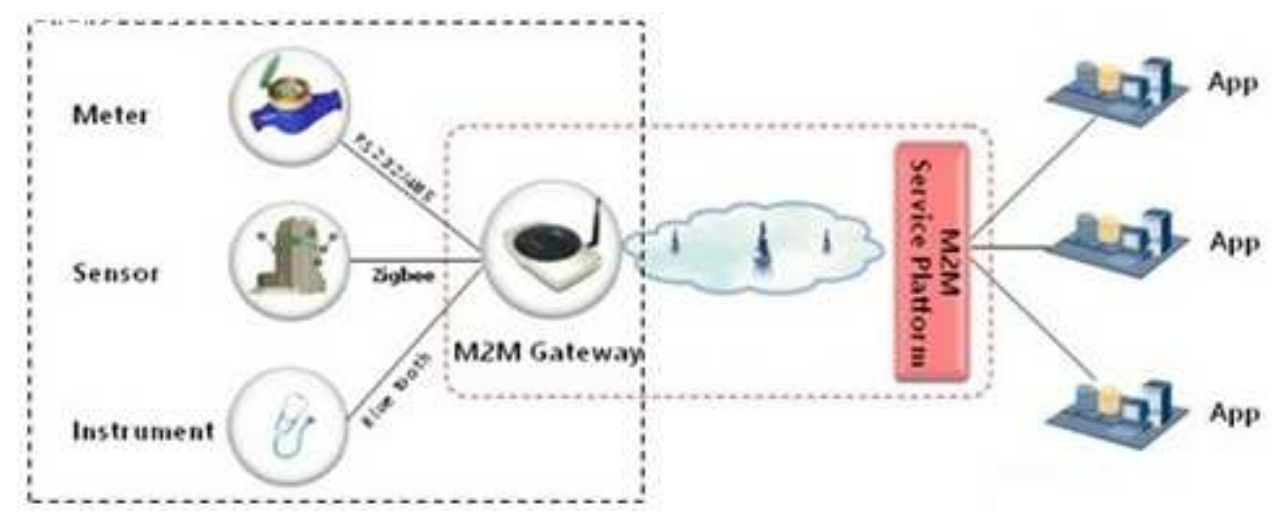

Figure 2. The communication architecture of digital hospital management

Intelligent wireless medical monitoring service is based on wireless LAN and RFID. Mobile hand held terminal data application is provided for front line staff through the use of intelligent "shift" touch the portable data terminal. Under the condition that the medical staff rounds or moves, it can carry on the data interaction with the hospital information system data center through the wifi network real-time on-line through the intelligent hand held data terminal and the nursing staff software. The medical staff obtain comprehensive medical data whenever and wherever possible by hand held data 
terminals, while patients can connect with the data center with RFID Bracelet in the hands, displaying the current inspection schedule, and to obtain comprehensive medical data. The settings of communication gateway of intelligent wireless medical monitoring service is shown as fig. 3 .

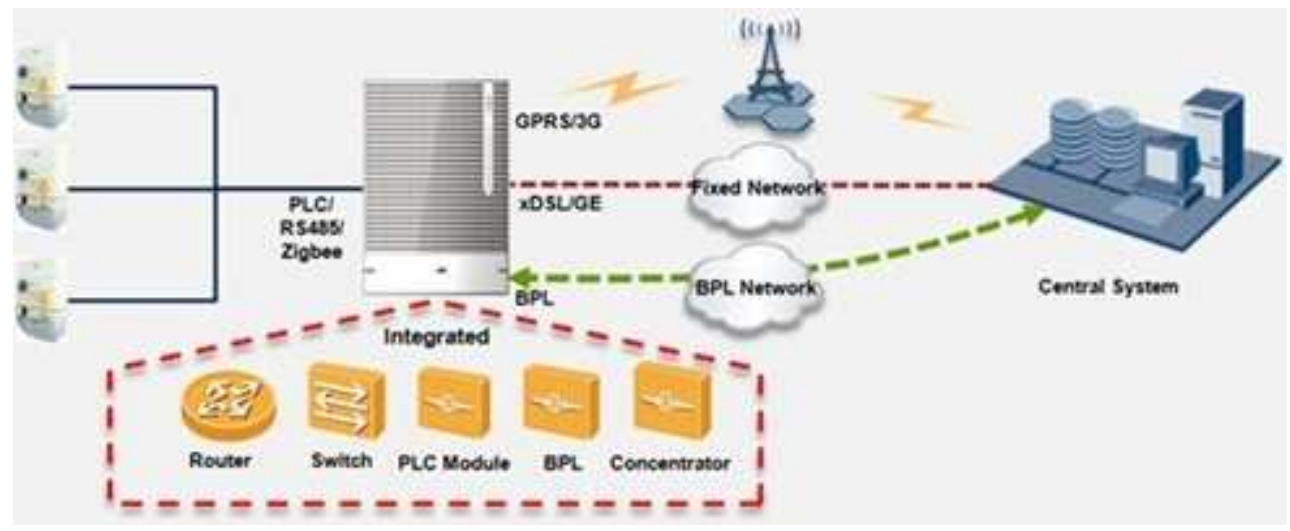

Figure 3. The settings of communication gateway of intelligent wireless medical monitoring service

At present, most of the families in the city has more than one household electronic diagnostic instrument, such as electronic blood pressure meter, electronic blood glucose meter, but the problem is that even if the patient measured the blood pressure and blood glucose data, doctors are still unable to obtain timely first-hand information through the sensor network technology. The doctor can understand in real time to the monitoring of physiological parameters patients. Medical Internet of things using embedded sensors and special software to make the community hospital doctors at home will be able to timely get the patient's various physiological parameters. This hidden sensor in electronic blood pressure meter and electronic blood glucose meter can be measured data in a timely manner through wireless transmission to the doctor's computer, and can be thousands of miles away in rural hospitals CT, MRI and other electronic diagnostic results using a sensor network and wireless transmission to a large medical center for expert consultation.

\section{Summary}

In the medical field, networking technology can be widely used, and can help the hospital to realize the intelligent management of people, material, equipment, support internal medical information, hospital equipment information, drug information, personnel information management, information processing, digital collection, storage, transmission, sharing, people and equipment, in order to realize visualization of material management, digitization, automation, improvement of medical safety and clinical efficiency, medical quality and patient safety. The application of the Internet of things in the wisdom medical treatment can solve the problem of resources loss.

\section{References}

[1] Z Qu, International Journal of Advancements in Computing Technology, Vol. 4 (2012) No.16, p.339.

[2] JA Guerrero-Ibanez, S Zeadally and J Contreras-Castillo, IEEE Wireless Communications, Vol. 22 (2015) No.6, p.122.

[3] S Luo and B Ren, Comput Methods Programs Biomed, Vol. 34 (2016) No.130, p.154.

[4] H Liu,J Wei,Z Xiong and G Wang, Advances in Information Sciences \& Service Sciences, Vol. 4 (2012) No.23, p.531. 
[5] Y Chen,S Zhao and Y Zhai,Journal of Chemical \& Pharmaceutical Research, Vol. 6 (2014) No.7, p.1676. 